\title{
Studies of Cavity Effects on Graphene Sheets via Molecular Dynamics
}

\author{
Avik Samanta, Qinghua Wang, Shaoping Xiao \\ 3131 Seamans Center, Department of Mechanical and Industrial Engineering, \\ The University of Iowa, Iowa City, Iowa 52242
}

\begin{abstract}
In this paper, the effects of cavities on graphene sheet failure were studied via molecular dynamics simulations. The graphene sheets with one centrally located cavity were simulated first. The Young's modulus and failure stress were calculated based on the simulated stress-strain curves. In addition, two or three cavities and their locations were also considered in this study. The Von Mises stress evolution indicated that the failure mechanism of graphene sheets with cavities were different from the one of a perfect graphene sheet.
\end{abstract}

Keywords: Graphene, cavity, molecular dynamics, fracture

\section{INTRODUCTION}

At nanoscale, a graphene sheet is in the form of a two-dimensional, honey-comb lattice in which one atom forms each vertex. The unique hexagonal molecular structure provides a basic structural element for other allotropes, including graphite, charcoal, carbon nanotubes and fullerenes. Most of them, especially carbon nanotubes [1-2], have extraordinary material and electrical properties and have been used to design novel nanoscale composites and devices [3-8].

Graphene also has great material and electrical properties. Its Young's modulus can be 1.0 tera-pascals [9], which is about five times stiffer than the strongest steel. In addition, there were lots of experimental researches on graphene focusing on its electrical properties. One of the most notable features on graphene transistors was the ability of continuously tuning the charge carriers from holes to electrons. The exceptional mobility of graphene, at low temperatures and high magnetic fields, was observed having the quantum effect for both electronics and holes [10-11]. Futher more, the roomtemperature thermal conductivity is high in graphene subject to size dependence [12]. Consequently, graphene is one of most commonly used inclusions for novel composite materials. However, the manufacturing of such composites requires not only that graphene sheets be produced on a sufficient scale but that they also be incorporated and homogeneously distributed into the matrix materials. Stankovich and co-workers [13] presented a general approach for the preparation of graphenepolymer composites via complete exfoliation of graphite and molecular-level dispersion of individual, chemically modified graphene sheets within polymer matrices.

It has been studied that defects had the effects on mechanical behaviors of most allotropes of carbon, including graphene sheets and carbon nanotubes. The defects include vacancies, metastable atoms, pentagon, heptagons, heterogeneous atoms, and discontinuities of planes etc [14, 15]. Among those type of defects, the Stone-Wales defects have received the most consideration. A Stone-Wales defect contains two pentagons and two heptagons instead of hexagons. Previous studies proposed that the Stone-Wales defects could result in the nucleation of a crack [16] although the failure stresses and strains were not significantly reduced [17]. Another type of defects, the vacancy defects, i.e. defects resulting from missing carbon atoms, have received considerable attention because the vacancy defects can severely reduce the strength of carbon nanostructures. It has been known such defects could be caused by ion irradiation, absorption of electrons, or carbon nanostructure fabrication processes. In the past few years, the continuum mechanics methods have been extensively used to study the mechanical properties [18] and fracture behavior [19] of graphene sheets. However, the continuum approximations are only applicable to study the mechanics of infinitely large nanosystems. In addition, it is difficult to investigate defect effects via continuum modeling and simulations. Consequently, as one of powerful numerical methods, molecular dynamics (MD) has 
been widely used to study mechanical behaviors of graphene sheets and carbon nanotubes at the nanoscale. It has been studied that vacancy defects play the most critical role in affecting the mechanical properties of graphene [20-21]. Mielke and co-workers [22] employed molecular mechanics and quantum mechanical calculations to study the role of vacancy defects in the fracture of carbon nanotubes under uniaxial tension. They found that vacancy defects can dramatically reduce the nanotube failure stresses. Xiao and Hou [23] studied facture of vacancy-defected nanotubes and their reinforced nanocomposites. They also adapted the spatial Poisson point process to study mechanical behaviors of carbon nanotubes with randomly located vacancy defects [24].

In this paper, more detailed analyses of the cavity effects on mechanics of graphene sheets is presented. The outline is described as below. After the introduction, the methodology is described in section 2. Results are discussed in section 3 followed by the conclusions.

\section{Methodology}

The problem definition is shown in Figure 1. The mechanical behavior of graphene sheets with and without a cavity was studied first using MD simulation. Then, various numbers and positions of cavities were considered in graphene sheets to provide a complete and detailed analysis of cavity effects on mechanics of graphene. The material properties, including Young's modulus and the failure stress, were also calculated for better understanding of the cavity effect.

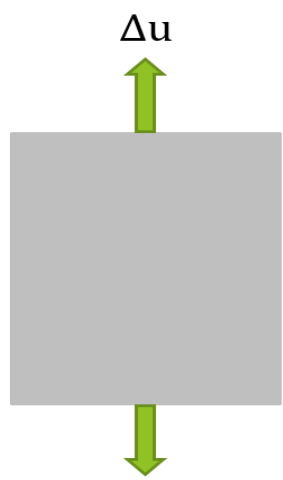

$\Delta \mathrm{u}$

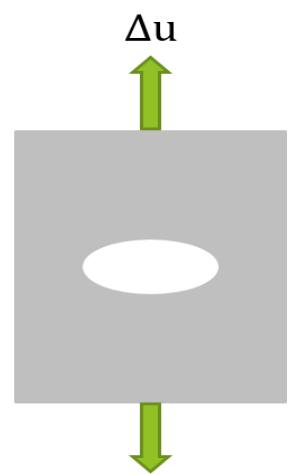

$\Delta \mathrm{u}$

Figure1. Problem definition and setup

An ensemble is a collection of all the possible states of a real system. The isothermal-isobaric (NPT) ensemble is used in this paper for all the simulations. The abbreviations $\mathrm{N}, \mathrm{P}$, and $\mathrm{T}$ stand for the number of atoms, the pressure and the temperature of the system, respectively. These quantities were kept constant during the MD simulations. In the MD simulation, the atoms or molecules in the simulated system follow the laws of classical mechanics. The motion of an atom, e.g. atom i, with mass $m_{i}$, is due to its interaction with other atoms in the system according to Newton's second law:

$m_{i} \vec{a}_{i}=\vec{f}_{i}=-\nabla W=-\frac{\partial W}{\partial \vec{r}_{i}}$

where $\vec{a}_{i}$ is the acceleration of atom $i$, and the interatomic force, $\vec{f}_{i}$, applied on atom $i$, is derived from the total potential energy, $\mathrm{W}$.

The MD simulations in this study were performed using the Large-scale Atomic Molecular Massively Parallel Simulator (LAMMPS), which is an open-source software written in C++ and developed at Sandia National Labs. The AtomEye and Techplot software provided a means to visualize LAMMPS output data and debug any problems in LAMMPS input scripts. To optimize performance, LAMMPS was implemented using the MPI message passing library for parallel processing.

In this paper, the adaptive intermolecular reactive empirical bond order (AIREBO) potential field was used to described interatomic interaction between carbon atoms in graphene as

$E^{A I R E B O}=\frac{1}{2} \sum_{i} \sum_{i \neq j}\left[E_{i j}^{R E B O}+E_{i j}^{L J}+\sum_{k \neq i, j} \sum_{l \neq i, j, k} E_{i j k l}^{\text {tors }}\right]$

where $E_{i j}^{R E B O}$ is the REBO part, which explains the bonded interaction between atoms; $E_{i j}^{L J}$ is Lennard-Jones potential that considers the non-bonded interactions between atoms; $E_{i j k l}^{\text {tors }}$ includes the torsional interactions between atoms into the total energy. It should be noted that the potential field of 
AIREBO was pre-built in with LAMMPS. The required additional reaction force field data for Carbon-Hydrogen-Oxygen (C-H-O) Systems are available on the website of NIST.

In each simulation, a simulation domain was defined first with 3D dimensions although only one layer of graphene sheet was simulated. Periodic boundary conditions were defined in all the three directions, and it is required by the NPT ensemble in LAMMPS. The temperature was maintained at the room temperature of $300 \mathrm{~K}$. Each simulation was conducted in two steps: a) self-balance or relaxation stage; b) deformation stage. A constant time step of $0.001 \mathrm{ps}$ was given in both stages in all the simulations.

The first stage of relaxation was assigned with a duration of $10 \mathrm{ps}$ for all the simulations. The simulated system would reach to the thermodynamic equilibrium state at the end of first stage. Once the second stage for deformation started, the simulation domain was elongated along the prescribed deformation direction, which is $\mathrm{y}$-axis in this study. A constant strain rate of $0.01 \mathrm{ps}^{-1}$ was given for all the simulations, and the deformation stage would last for $50 \mathrm{ps}$. Therefore, the total strain (deformation) is 50\% which is higher than reported failure strain of graphene. Pressure controls were applied at the boundaries in $\mathrm{x}$ - and $\mathrm{z}$-directions. This allows the simulation domain to shrink in these two directions so that the pressure could be kept as a constant required by the NPT ensemble.

\section{RESULTS AND DISCUSSIONS}

A graphene sheet containing a total number of 855 carbon atoms was employed in this paper to study cavity effects on mechanics of graphene sheets. The atom numbers in $\mathrm{x}$ - and $\mathrm{y}$-axis are $19 \times 45$. A graphene sheet without cavity was simulated first, and its failure behavior is shown in Figure 2 . Then, a graphene sheet with a single cavity was considered, and the circular cavity was prescribed at the center of the simulation domain. The diameter of the cavity is $1 / 3$ of the width of the simulation domain. The simulation results were compared to the ones from a perfect graphene sheet. Figure 3 shows the domain evolution as well as the failure of the graphene sheet with one cavity. In Figure 3, greater shear strains can be seen on the both sides of the cavity, where the crack was initiated and led to the catastrophic failure. In addition, stress concentration is expected at the critical location with greater shear strains.

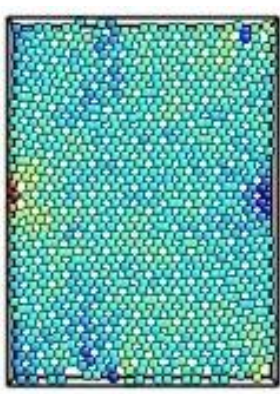

(a) time $=0 \mathrm{ps}$

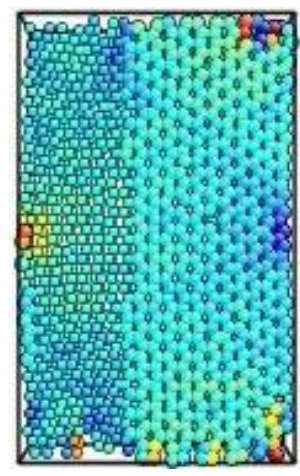

(b) time $=15 \mathrm{ps}$

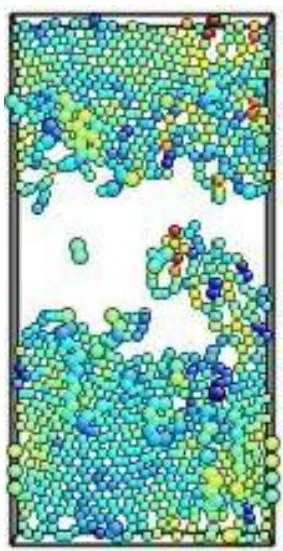

(c) time $=36 \mathrm{ps}$

Figure2. Fracture behavior of a graphene sheet without cavity

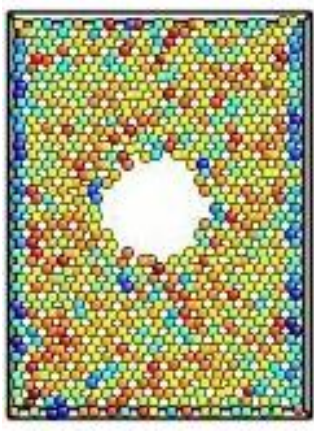

(a) time $=0 \mathrm{ps}$

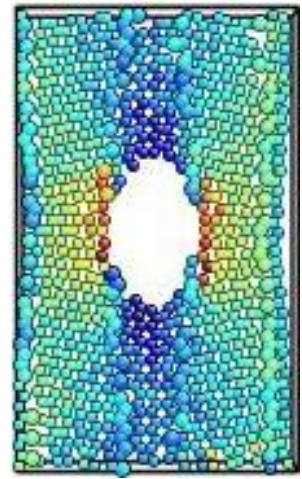

(b) time $=15 \mathrm{ps}$

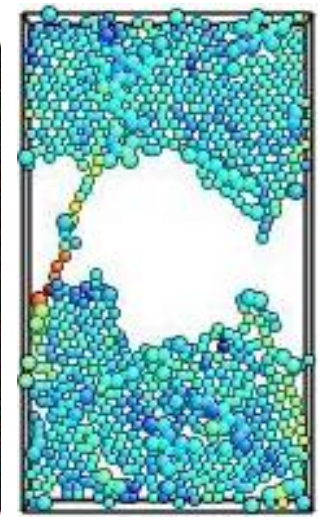

(c) time $=23 \mathrm{ps}$

Figure3. Fracture behavior of a graphene sheet with one cavity 
Figure 4 shows the total potential and the stress at various strains during the simulation. It can be seen that the graphene sheet with a cavity failed at $23 \%$ strain, which is smaller than the $36 \%$ failure strain of the graphene sheet without cavity. In addition, the Young's modulus can be calculated based on the slope of stress-strain curves. The estimated Young's modulus during the elastic stage are $0.732 \mathrm{TPa}$ and $0.523 \mathrm{TPa}$ for the perfect graphene sheet and the defected graphene sheet respectively.

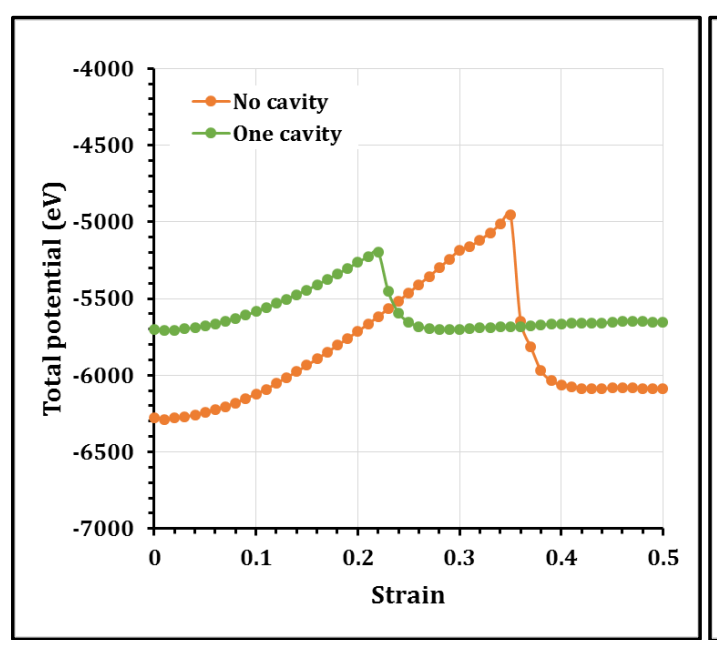

(a) potential energy

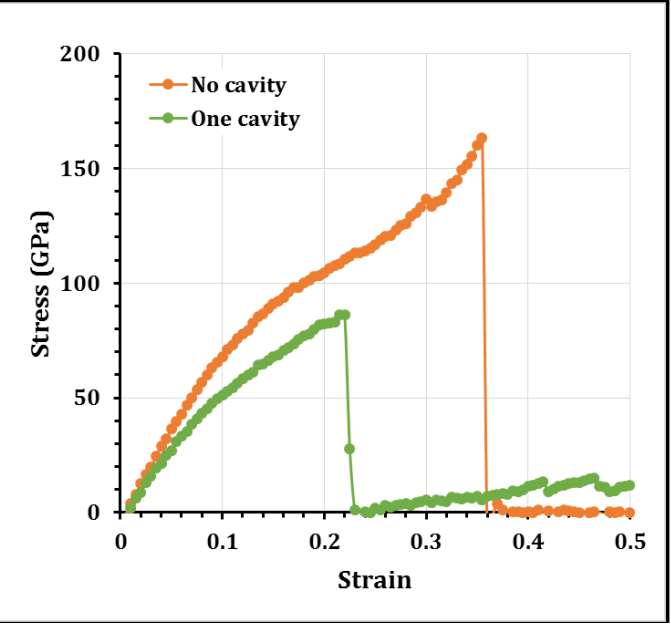

(b) stress-strain behavior

Figure4. Effect of cavity on potential energy and stress-strain behavior

The number of cavities affects the mechanical behavior of graphene sheets as well. Graphene sheets with one or two cavities were considered in this study. The results were compared to the one from the perfect graphene as shown in Figure 5. It can be seen that the graphene sheet with more cavities failed earlier. In addition, the Young's modulus decreases when the number of cavities increases.

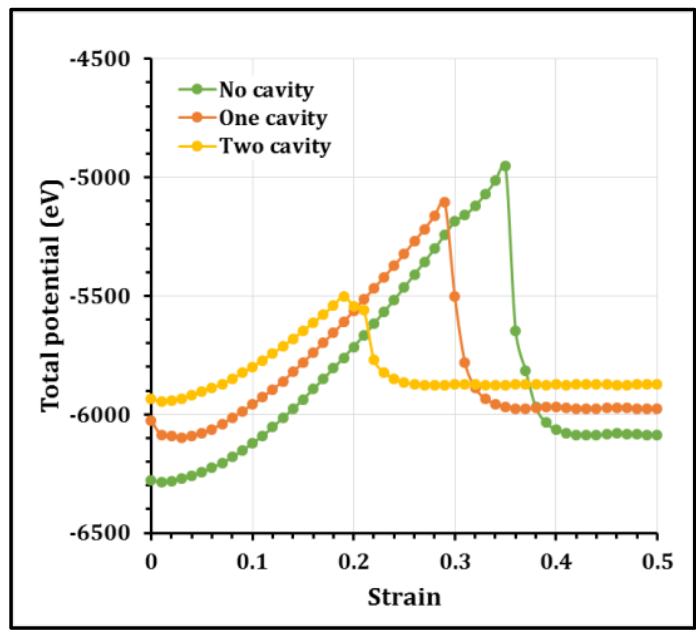

(a) potential energy

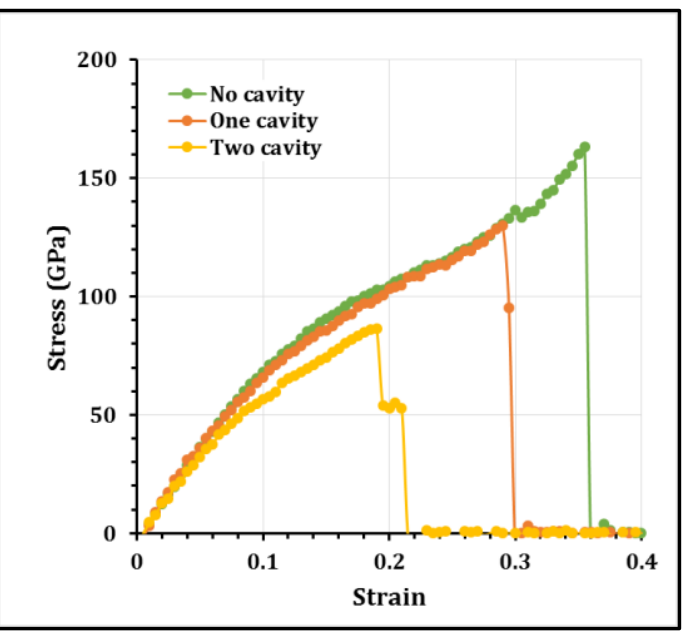

(b) stress-strain behavior

Figure5. Effect of cavity numbers on potential energy and stress-strain behavior

It shall be noted that the failure patterns are different between graphene sheets with one or two cavities. In the graphene sheet with two cavities, as shown in Figure 6, the failure occurred between the cavities at first, and two cavities emerged as a big one. Then, the catastrophic failure occurred. In addition, the phenomena can be clearly explained via the comparison of Von Mises stress evolutions among those three graphene sheets as shown in Figure 7 and Figure 8. In the graphene sheet without cavity, the stress continuously increased fairly uniform over the whole simulation domain. However, in the graphene sheet with one cavity, the stress concentration occurred at the both ends of the single cavity, where the cracks were initiated and the catastrophic failure occurred. In the graphene sheet with two cavities, Von Mises stress concentration initially happened between the two cavities. After two cavities emerged as a big one due to crack initiation and propagation, stress concentration occurred at the two side edges prior to catastrophic failure as shown in Figure 8. 


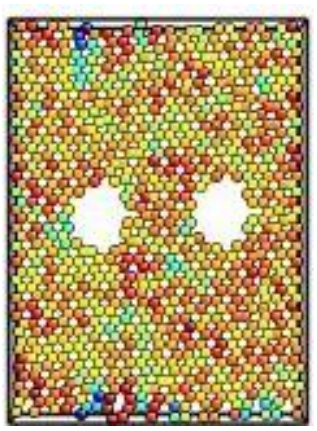

(a) time $=0 \mathrm{ps}$

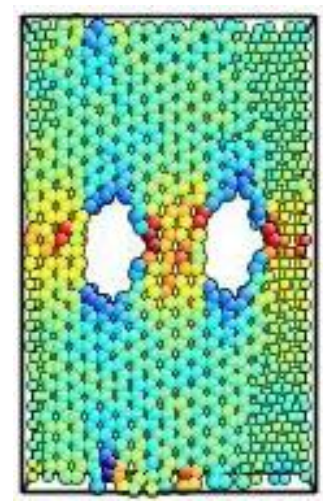

(b) time $=15 \mathrm{ps}$

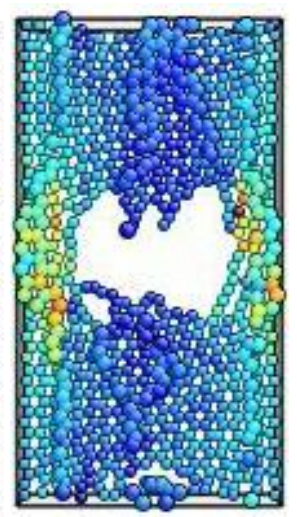

(c) time $=20 \mathrm{ps}$

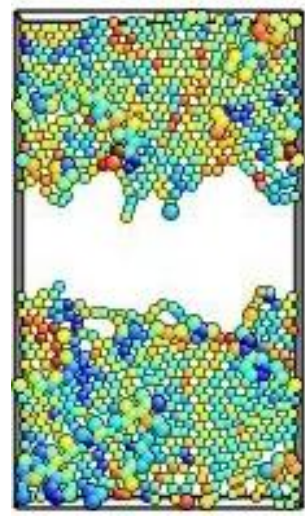

(d) time $=22 \mathrm{ps}$

Figure6. Fracture behavior of a graphene sheet with two cavities in horizontal position

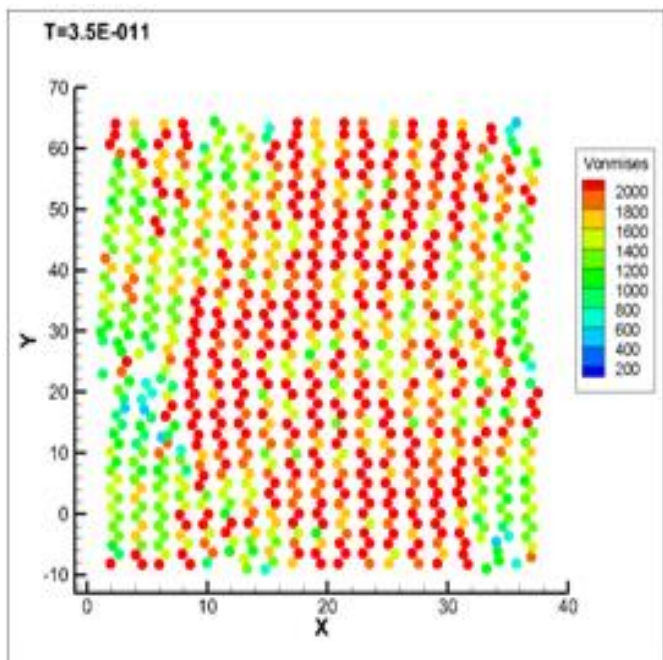

(a) graphene sheet without cavity

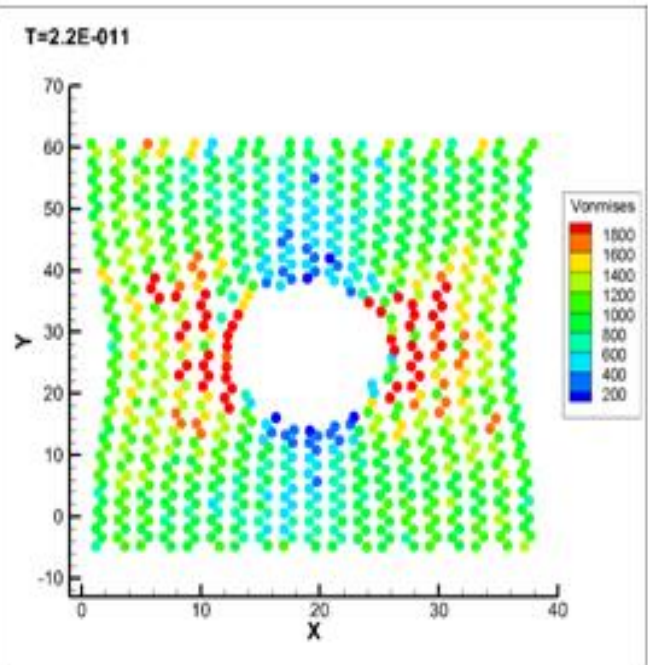

(b) graphene sheet with one cavity

Figure7. Von-Mises stress distribution before graphene sheet failure

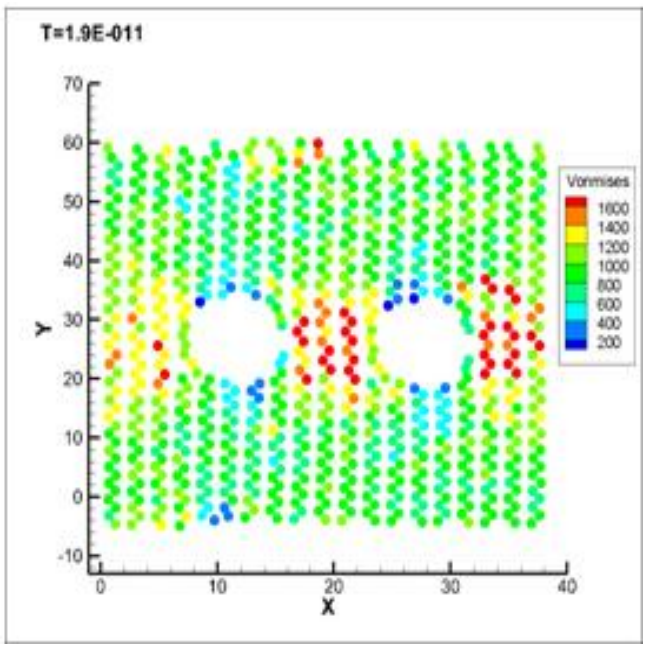

(a) before failure between two cavities

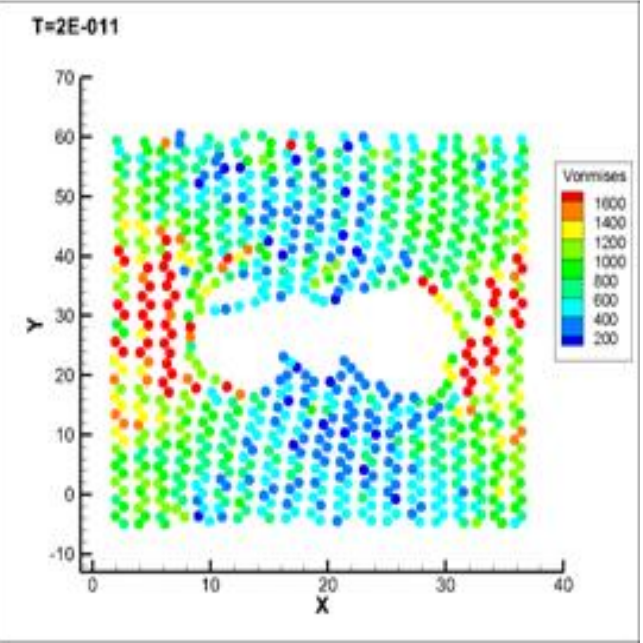

(b) before catastrophic failure

Figure8. Von-Mises stress distribution on the graphene sheet with two cavities in horizontal position

In the above study, two cavities were placed in the horizontal position. To investigate the effect of cavity location on the failure of graphene sheets, two other graphene sheets are considered. One graphene sheet has two cavities in the vertical position while the other has three cavities in zigzag position. The potential energies and stress-strain relationships are plotted in Figure 9 and compared to a perfect graphene sheet. It can be seen that three cavity-defected graphene sheets have the similar Young's moduli, which are smaller than the one of a perfect graphene sheet. 


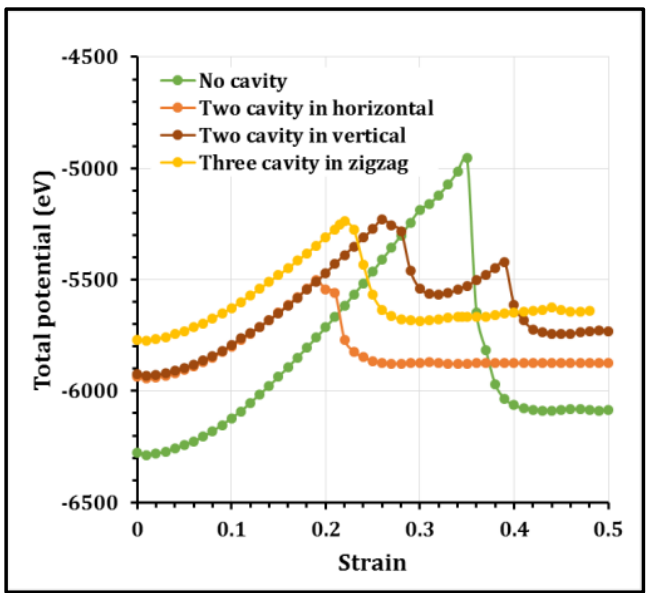

(a) potential energy

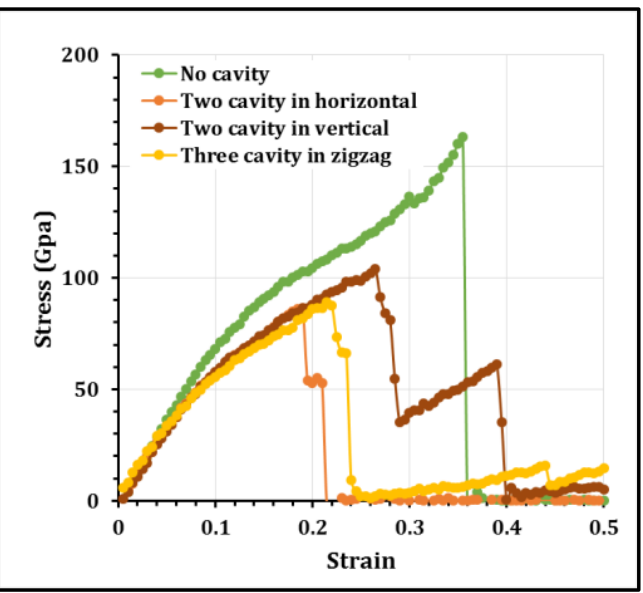

(b) stress-strain behavior

Figure9. Effect of cavity position on potential energy and stress-strain behavior

However, their failure stresses are different. The graphene sheet with two cavities in vertical position has the highest failure stress while the sheet with two cavities in horizontal position has the lowest failure stress. Especially in the graphene sheet with two cavities in vertical position, the failure first initiate with two cracks at the outer edges which are close to the cavities as shown in Figure 10. This leads to the first drop in the stress strain curve in Figure 9(b) at the strain of 27\%. The catastrophic failure occurred at much higher strain, $40 \%$, when two cracks propagated and merged as one.

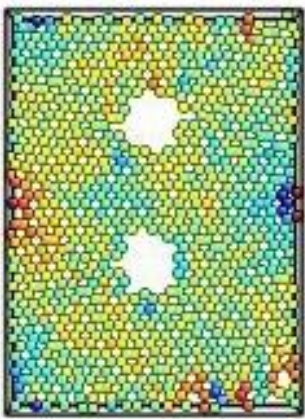

(a) time $=0 \mathrm{ps}$

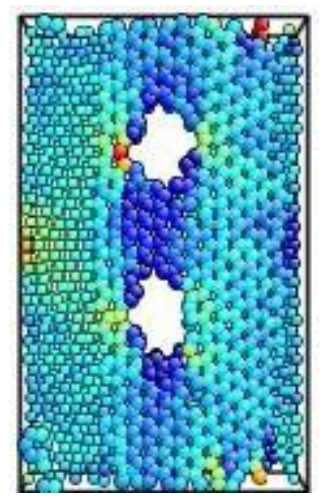

(b) time $=15 \mathrm{ps}$

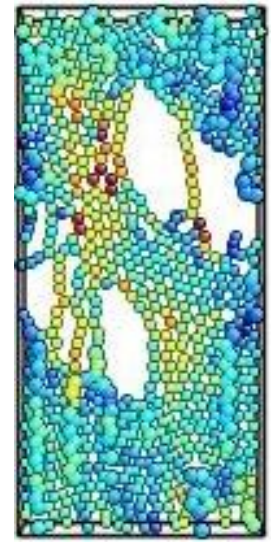

(c) time $=30 \mathrm{ps}$

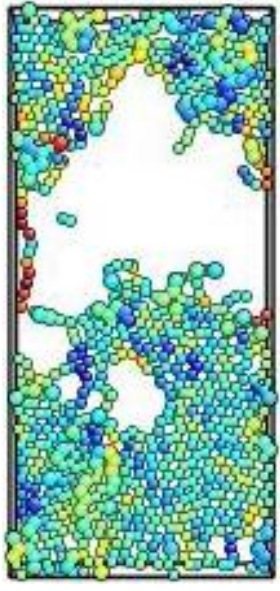

(d) time $=40 \mathrm{ps}$

Figure10. Fracture behavior of a graphene sheet with two cavities in vertical position

\section{Conclusions}

Molecular dynamics simulations were conducted in this paper to study the effects of cavities on mechanics of graphene sheets. It has been observed that the graphene sheets with cavities have lower Young's modulus and failure stresses, compared to the perfect graphene sheet. Especially, the graphene sheet with more cavities has lower modulus and failure stress. The stress concentration usually occurred around the cavities before the catastrophic failure occurred. It shall be noted that the failure mechanisms between defected graphene sheets were different. In the graphene sheet with two cavities in horizontal position, a crack initiated between cavities first. The catastrophic failure occurred after two cavities emerged to a big one. In the graphene sheet with two cavities in vertical position, two cracks initiated by two cavities simultaneously before the catastrophic failure occurred. It has been found that the graphene sheet with two cavities in vertical position has higher failure stress than the one with two cavities in horizontal position.

\section{REFERENCES}

[1] Iijima S., Helical microtubules of graphitic carbon, Nature, 354, 56 (1991).

[2] Popov V. N., Carbon nanotubes: properties and application, Mat. Sci. Eng. R - Reports, 43(3), 61 (2004). 
[3] Xiao, S. P., Han, R. and Hou, W. Y., Spin in carbon nanotube-based oscillators, Int. J. Nanosci., 5, 74 (2006).

[4] Xiao S. P., Andersen D. R., Han R. and Hou W. Y., Studies of carbon nanotube-based oscillators using molecular dynamics, Int. J. Comp. Theo. Nanosci., 3, 142 (2006).

[5] Xiao, S. P. and Hou, W. Y., Studies of nanotube-based aluminum composites using the bridging domain coupling method, Int. J. Multi. Comp. Eng., 5, 447 (2007).

[6] Xiao, S. P. and Hou, W. Y., Studies of nanotube-based resonant oscillators via multiscale modeling and simulation, Phys. Rev. B, 75125414 (2007).

[7] Xiao, S. P. and Hou, W. Y., Multiscale modeling and simulation of nanotube-based torsional oscillators, Nano. Res. Lett., 2, 54 (2007).

[8] Xiao, S. P., Andersen, D. R. and Yang, W. X., Design and analysis of nanotube-based memory cells, Nano. Res. Lett., 3, 416 (2008).

[9] Lee, C. G., Wei, X. D., Kysar, J. W. and Hone, J., Measurement of the elastic properties and intrinsic strength of monolyaer graphene, Sci., 321, 385 (2008).

[10] Novoselov, K. S., Geim, A. K., Morozov, S. V., Jiang, D., Katsnelson, M.I., Grigorieva, I. V., Dubonos, S. V. and Firsov, A. A., Two-dimensional gas of massless Dirac fermions in graphene, Nature, 438, 197 (2005).

[11] Zhang, Y. B., Tan, Y. W., Stormer, H. L. and Kim P., Experimental observation of the quantum Hall effect and Berry's phase in graphene, Nature, 438, 201 (2005).

[12] Balandin, A. A., Thermal properties of graphene and nanostructured carbon materials. Nature Mat., 10, 569 (2011).

[13] Stankovich, S., Dikin, D. A., Dommett, G. H. B., Kohlhaas, K. M., Zimney, E. J., Stach, E. A., Piner R. D., Nguyen, S. T. and Ruoff, R. S., Graphene-based composite materials, Nature, 442, 282 (2006).

[14] Banhart, F., Irradiation effects in carbon nanostructures, Rep. Prog. Phys., 62, 1181 (1999).

[15] Iijima, S., Ichihashi, T., Ando, Y., Pentagons, heptagons and negative curvature in graphite microtubule growth, Nature, 356, 776 (1992).

[16] Yakobson, B. I., Mechanical relaxation and intramolecular plasticity in carbon nanotubes", Appl. Phys. Lett., 72(8), 918 (1998).

[17] Troya, D, Mielke, S. L., and Schatz, G. C., Carbon nanotube fracture - differences between quantum mechanical mechanisms and those of empirical potentials, Chem. Phys. Lett., 382, 133 (2003).

[18] Shokrieh, M. M., and Rafiee, R., Prediction of Young's modulus of graphene sheets and carbon nanotubes using nanoscale continuum mechanics approach, Mat. Design., 31(2), 790 (2010).

[19] Xu, M., Tabarraei, A., Paci, J. T., Oswald, J., and Belytschko, T., A coupled quantum/continuum mechanics study of graphene fracture, Int. J. Frac., 173(2), 163 (2012).

[20] Dewapriya, M. A. N., and Rajapakse, R. K. N. D., Effects of Free Edges and Vacancy Defects on the Mechanical Properties of Graphene, Conference Proceeding, 14th IEEE Int. Conference on Nanotechnology, Pp. 908-912 (2014).

[21] Ito, A., and Okamoto, S., Mechanical Properties of Vacancy-containing Graphene and Graphite using Molecular Dynamics Simulations, Conference Proceeding, Int. MultiConference of Engineers and Computer Scientists, (2012).

[22] Mielke, S. L., Troya, D., Zhang, S., Li, J., Xiao, S., Car, R., Ruoff, R. S., Schatz, G. C., and Belytschko, T., The role of vacancy defects and holes in the fracture of carbon nanotubes, Chem. Phys. Lett., 390, 413 (2004).

[23] Xiao, S. P. and Hou, W. Y., Fracture of vacancy-defected carbon nanotubes and their embedded nanocomposites, Phys. Rev. B, 73, 115406 (2006).

[24] Hou, W. Y. and Xiao, S. P., Mechanical behaviors of carbon nanotubes with randomly located vacancy defects, J. Nanosci. Nanotech., 7, 1 (2007). 


\section{AUTHORS' BIOGRAPHY}

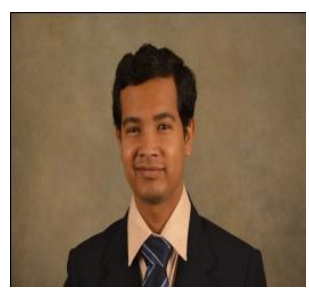

Avik Samanta, received the B.E. degree in Production Engineering from Jadavpur University, Kolkata, India, in 2009 and M.Tech. degree in Mechanical Engineering from Indian Institute of Technology Bombay, Mumbai, India, in 2012.

Currently he is a PhD Scholar with the Department of Mechanical and Industrial Engineering, University of Iowa, Iowa City, IA, USA. His research interests include laser-based materials processing, physics-based process modeling, micro-structure modeling, multi-scale modeling, advanced joining process of similar and dissimilar materials and hybrid manufacturing processes

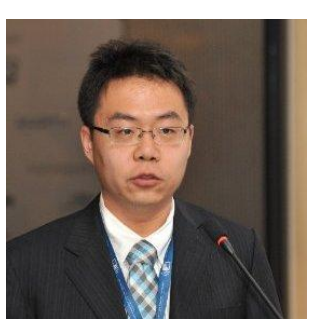

Qinghua Wang, received the B.E. degree in Materials Processing and Control Engineering from Huazhong University of Science and Technology, Wuhan, China, in 2013 and M. Eng. degree in Mechanical Engineering from University of Wisconsin-Madison, Madison, USA, in 2015. Currently he is a $\mathrm{PhD}$ student with the Department of Mechanical and Industrial Engineering, University of Iowa, Iowa City, IA, USA. His research interests include laser materials processing and microstructure simulation for advanced manufacturing processes.

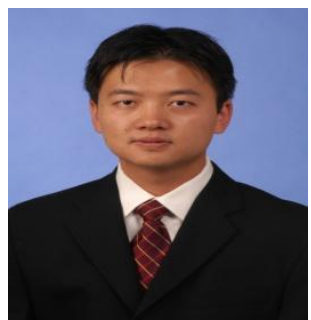

Shaoping Xiao, is an associate professor in the Department of Mechanical and Industrial Engineering at The University of Iowa. He was graduated from Northwestern University with a $\mathrm{PhD}$ degree in mechanical engineering in 2003 before joining The University of Iowa. His group's research interests include finite element method, meshfree particle methods, multiscale modeling and simulation. 